\title{
Use of Silence as an Altered Approach for Speaker Recognition
}

\author{
Rupali Pawar ${ }^{1}$, R. M. Jalnekar ${ }^{2}$ and J. S. Chitode ${ }^{3}$ \\ ${ }^{1}$ Research Scholar, Vishwakarma Institute of Technology, Pune \\ ${ }^{2}$ Director \& Professor, Vishwakarma Institute of Technology, Pune \\ ${ }^{3}$ Professor, E \&TC Department, Vishwakarma Institute of Technology, Pune \\ ${ }^{1}$ rvspawar@rediffmail.com, ${ }^{2}$ director@vit.edu, ${ }^{3}$ j.chitode@gmail.coml
}

\begin{abstract}
Speaker recognition is an important application of Speech Signal Processing and has been used in public safety, authenticating users for important financial transactions, access control systems and many more. The conventional approach in speaker identification and speaker verification has been to remove the silence from the recorded speech signal and further extract the significant features from the residual signal for recognition. This paper presents an alternative approach and puts forth the experimental results of obtaining silence as a parameter to check if the pattern of pauses/silence for train and test files recorded for individual speaker match. The paper emphasizes the approach in which a paragraph is recorded for 8 speakers and used as train files. The duration of silencelpauses of the speaker in a paragraph are obtained. This silence obtained is compared with the silence obtained from test file the matching of pattern of the silences decides the identity of the speaker.
\end{abstract}

Keywords: Framing, intra speaker variability, inter speaker variability, normalization, threshold

\section{Introduction}

The Speaker recognition system has four phases Analysis, Feature Extraction, Recognition and Testing. In the speaker Recognition system the database used for processing can be standard or recorded. For a robust Speaker Recognition system the database should be free from inter speaker and intra speaker variability, as these are crucial parameters and their presence may affect the performance of the system. The intra speaker variation is due to variable speaking rate, changing emotions or other mental variables, and also due to environment noise, emotional state of the speaker at the time of recording etc. [1]. The inter speaker variability is variability in the speech signal due to individual variations in the speech of the speaker. This can be due to variations in excitation source, length of the vocal cord and movement of articulators. The conventional Speaker Recognition System extracts features like MFCC, pitch, duration as features for identification after the pre-emphasis stage during which the speech signal is made noise and silence free. This paper puts forth an approach where silence is obtained from the speech signal of a speaker and saved in the train folder which is further analyzed to check if the pattern of silence for the speaker remains the same any number of times it is recorded.

\section{Implementation}

To implement the above approach database of 8 speakers (7 Females, 1 male) is used. A paragraph of approximately $50 \mathrm{sec}$ is recorded in a .wav file format and stored in the train folder. The same paragraph is again recorded for the above 8 speakers and stored in the test folder. The paragraph is divided into three parts as Initial paragraph, Intermediate 
paragraph and End paragraph of around $16 \mathrm{sec}$ each. The purpose or motive behind this is to capture the naturalness of the speaker. It is assumed that maximum spontaneity might be captured better in the intermediate part as a speaker might be conscious while recording the initial few lines and may be in a rush to complete the paragraph towards the end.

The recorded speech signals is framed in chunk of $20 \mathrm{mS}$ to $30 \mathrm{mS}$ and s analyzed to obtain silence in each frame. The overall speech is analyzed to demark silence, a threshold is fixed, the part of speech signal lying between this threshold values is marked as silence and the duration of this silence is captured in number of samples. Figure 1 depicts the speech signal of speaker 1, Intermediate paragraph and silence obtained from the same after applying threshold for test and train files respectively.
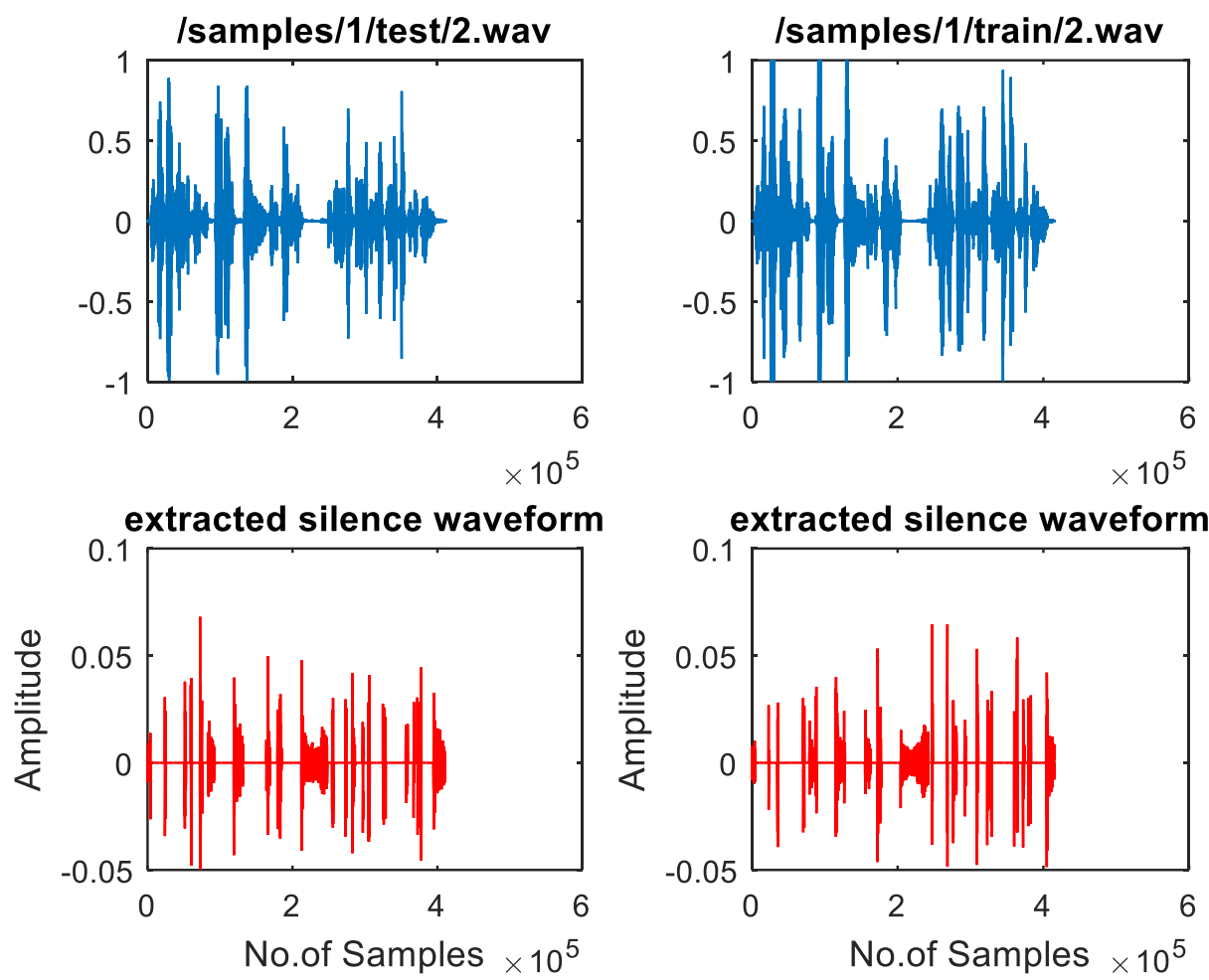

Figure 1. Speech Signal (blue) and Silence Extracted (red) for Test and Train Files of Intermediate Paragraph of Speaker 1

\subsection{Algorithm}

1. Record the data at different instances and save as train and test file

2. Frame the speech signal into chunks of $10-30 \mathrm{mS}$

3. To find the duration of pauses/silence occurring at various instances in the sentence, compare the amplitude of speech signal with positive and negative threshold value. ( chosen by permutation combination for the recorded speech)

4. Normalize the silence duration noted.

5. Correlate the normalized data to find the match in the pattern of silence for each Speaker

\section{Experimentation}

As shown in Figure 1 the speech signal is recorded and it is observed that the sentence in each paragraph has certain pauses. Instead of removing this silence, the 
speech signal is framed, and the silence duration which is in between the threshold values is obtained and noted. The duration of silence obtained for each instance is recorded for both the test and train file of every speaker for each initial, intermediate and end paragraph as shown in the Table 1 below. The duration of silence/pauses obtained has large variation in the value and hence the data is normalized. The normalized values of train and test data are compared to find the similarity in the two signals thereby allowing us to compare whether the pauses are of similar pattern for a speaker. Certain observations are put forth after plotting graph to compare the normalized train and test data for respective paragraph recorded for all the speakers, as depicted in Figure $2 \mathrm{a} \& 2 \mathrm{~b}$

1. The pattern may shift to certain extent during different recording sessions.

2. The pattern of silence for intermediate paragraph show better similarity compared to Initial and End paragraph.

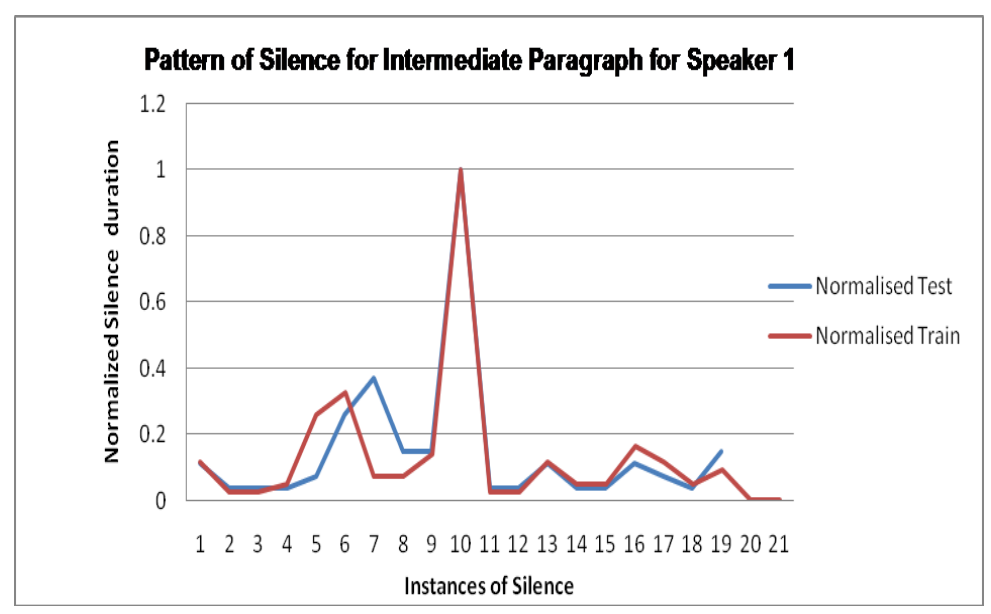

Figure 2a: Pattern of Silence for Intermediate Paragraph for Speaker 1 Normalised Values of Silence Duration ( $y$-axis) Vs Instances of Silence (x-Axis)

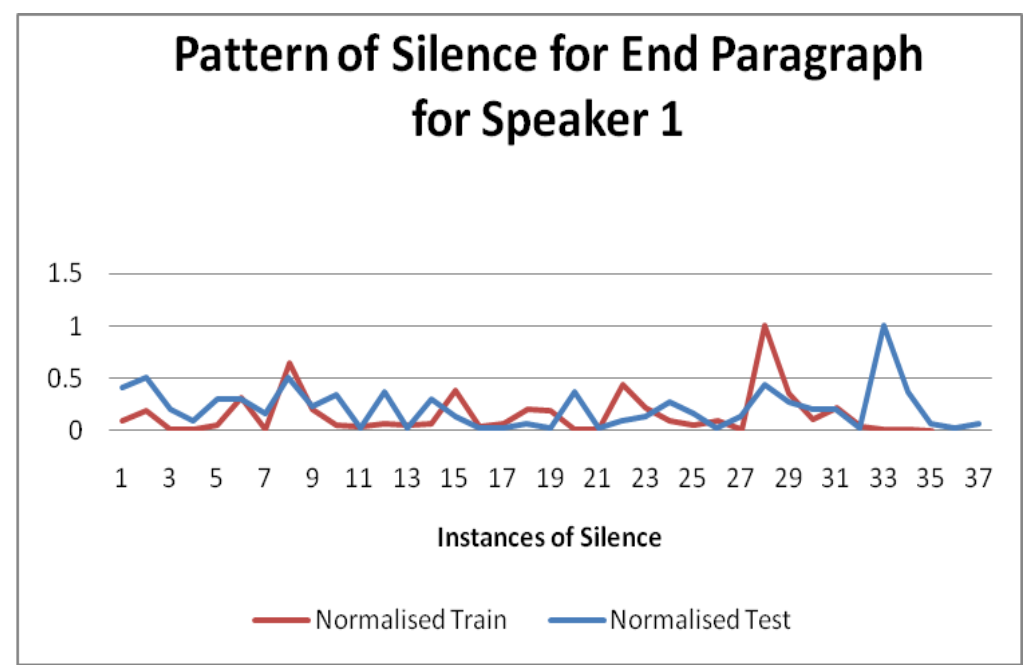

Figure 2b: Pattern of Silence for End Paragraph for Speaker 1 Normalised values of silence duration ( $y$-axis) Vs Instances of Silence (x-axis) 
Table 1. Test and Train Data Recorded and Normalized for Initial, Intermediate and End Paragraph for Speaker 1

\begin{tabular}{|c|c|c|c|c|c|c|c|c|c|c|c|}
\hline \multicolumn{12}{|c|}{ Speaker 1} \\
\hline \multicolumn{4}{|c|}{ Start Paragraph } & \multicolumn{4}{|c|}{ Intermediate Paragraph } & \multicolumn{4}{|c|}{ End Paragraph } \\
\hline Test & $\begin{array}{l}\text { Normali } \\
\text { zed Test }\end{array}$ & Train & $\begin{array}{l}\text { Normalize } \\
\text { d Train }\end{array}$ & Test & $\begin{array}{l}\text { Normalize } \\
\text { d Test }\end{array}$ & Train & $\begin{array}{l}\text { Normali } \\
\text { zed } \\
\text { Train }\end{array}$ & Test & $\begin{array}{l}\text { Normalize } \\
\text { d Test }\end{array}$ & Train & $\begin{array}{l}\text { Normaliz } \\
\text { ed Train }\end{array}$ \\
\hline 58080 & 1 & 89760 & 1 & 3960 & 0.111 & 4400 & 0.116 & 15840 & 0.414 & 4400 & 0.094 \\
\hline 1320 & 0.023 & 880 & 0.01 & 1320 & 0.037 & 880 & 0.023 & 19800 & 0.517 & 8800 & 0.189 \\
\hline 1320 & 0.023 & 3520 & 0.039 & 1320 & 0.037 & 880 & 0.023 & 7920 & 0.207 & 880 & 0.019 \\
\hline 2640 & 0.045 & 880 & 0.01 & 1320 & 0.037 & 1760 & 0.047 & 3960 & 0.103 & 880 & 0.019 \\
\hline 1320 & 0.023 & 13200 & 0.147 & 2640 & 0.074 & 9680 & 0.256 & 11880 & 0.31 & 2640 & 0.057 \\
\hline 10560 & 0.182 & 880 & 0.01 & 9240 & 0.259 & $\begin{array}{l}1232 \\
0\end{array}$ & 0.326 & 11880 & 0.31 & 14960 & 0.321 \\
\hline 3960 & 0.068 & 2640 & 0.029 & $\begin{array}{l}1320 \\
0\end{array}$ & 0.37 & 2640 & 0.07 & 6600 & 0.172 & 880 & 0.019 \\
\hline 1320 & 0.023 & 2640 & 0.029 & 5280 & 0.148 & 2640 & 0.07 & 19800 & 0.517 & 29920 & 0.642 \\
\hline 3960 & 0.068 & 2640 & 0.029 & 5280 & 0.148 & 5280 & 0.14 & 9240 & 0.241 & 9680 & 0.208 \\
\hline 1320 & 0.023 & 2640 & 0.029 & $\begin{array}{l}3564 \\
0\end{array}$ & 1 & $\begin{array}{l}3784 \\
0\end{array}$ & 1 & 13200 & 0.345 & 2640 & 0.057 \\
\hline 1320 & 0.023 & 31680 & 0.353 & 1320 & 0.037 & 880 & 0.023 & 1320 & 0.034 & 1760 & 0.038 \\
\hline 2640 & 0.045 & 880 & 0.01 & 1320 & 0.037 & 880 & 0.023 & 14520 & 0.379 & 3520 & 0.075 \\
\hline 3960 & 0.068 & 2640 & 0.029 & 3960 & 0.111 & 4400 & 0.116 & 1320 & 0.034 & 2640 & 0.057 \\
\hline 1320 & 0.023 & 9680 & 0.108 & 1320 & 0.037 & 1760 & 0.047 & 11880 & 0.31 & 3520 & 0.075 \\
\hline 31680 & 0.545 & 1760 & 0.02 & 1320 & 0.037 & 1760 & 0.047 & 5280 & 0.138 & 17600 & 0.377 \\
\hline 13200 & 0.227 & 3520 & 0.039 & 3960 & 0.111 & 6160 & 0.163 & 1320 & 0.034 & 1760 & 0.038 \\
\hline 3960 & 0.068 & 2640 & 0.029 & 2640 & 0.074 & 4400 & 0.116 & 1320 & 0.034 & 3520 & 0.075 \\
\hline 1320 & 0.023 & 11440 & 0.127 & 1320 & 0.037 & 1760 & 0.047 & 2640 & 0.069 & 9680 & 0.208 \\
\hline 1320 & 0.023 & 1760 & 0.02 & 5280 & 0.148 & 3520 & 0.093 & 1320 & 0.034 & 8800 & 0.189 \\
\hline 10560 & 0.182 & 3520 & 0.039 & 0 & 0 & 0 & 0 & 14520 & 0.379 & 880 & 0.019 \\
\hline 1320 & 0.023 & 7040 & 0.078 & 0 & 0 & 0 & 0 & 1320 & 0.034 & 880 & 0.019 \\
\hline 3960 & 0.068 & 1760 & 0.02 & 0 & 0 & 0 & 0 & 3960 & 0.103 & 20240 & 0.434 \\
\hline 9240 & 0.159 & 34320 & 0.382 & 0 & 0 & 0 & 0 & 5280 & 0.138 & 10560 & 0.226 \\
\hline 1320 & 0.023 & 2640 & 0.029 & 0 & 0 & 0 & 0 & 10560 & 0.276 & 4400 & 0.094 \\
\hline 3960 & 0.068 & 13200 & 0.147 & 0 & 0 & 0 & 0 & 6600 & 0.172 & 2640 & 0.057 \\
\hline 1320 & 0.023 & 3520 & 0.039 & 0 & 0 & 0 & 0 & 1320 & 0.034 & 4400 & 0.094 \\
\hline 34320 & 0.591 & 2640 & 0.029 & 0 & 0 & 0 & 0 & 5280 & 0.138 & 880 & 0.019 \\
\hline 2640 & 0.045 & 2640 & 0.029 & 0 & 0 & 0 & 0 & 17160 & 0.448 & 46640 & 1 \\
\hline 9240 & 0.159 & 0 & 0 & 0 & 0 & 0 & 0 & 10560 & 0.276 & 16720 & 0.358 \\
\hline 2640 & 0.045 & 0 & 0 & 0 & 0 & 0 & 0 & 7920 & 0.207 & 5280 & 0.113 \\
\hline 2640 & 0.045 & 0 & 0 & 0 & 0 & 0 & 0 & 7920 & 0.207 & 10560 & 0.226 \\
\hline 0 & 0 & 0 & 0 & 0 & 0 & 0 & 0 & 1320 & 0.034 & 1760 & 0.038 \\
\hline 0 & 0 & 0 & 0 & 0 & 0 & 0 & 0 & 38280 & 1 & 880 & 0.019 \\
\hline 0 & 0 & 0 & 0 & 0 & 0 & 0 & 0 & 14520 & 0.379 & 880 & 0.019 \\
\hline 0 & 0 & 0 & 0 & 0 & 0 & 0 & 0 & 2640 & 0.069 & 0 & 0 \\
\hline 0 & 0 & 0 & 0 & 0 & 0 & 0 & 0 & 1320 & 0.034 & 0 & 0 \\
\hline 0 & 0 & 0 & 0 & 0 & 0 & 0 & 0 & 2640 & 0.069 & 0 & 0 \\
\hline
\end{tabular}


Table 1 depicts silence samples from Test and Train data recorded and normalized for Initial, Intermediate and End paragraph for speaker 1. The graph of the normalized train and test data for all the speakers for initial, intermediate and end part of the paragraph after experimentation show that the silence pattern in intermediate and end paragraph have better matching compared to the initial paragraph.

Figure 2 a \& b represent the graph of normalized silence sample values of Initial paragraph and end paragraph for speaker $1 \mathrm{Vs}$ the instances of silence is plotted. The comparison shows that silence in intermediate paragraph have better matching results compared to the end paragraph.

It is observed that Intermediate paragraph shows better correlation values for normalized Train and Test samples compared to end paragraph.

\section{Testing \& Results}

The correlation of the normalized silence values for each speaker in the train and Test folders are depicted in the Table 2

\section{Table 2: Correlation between Normalized Silence Sample Values of Test and} Train Dta for Initial, Intermediate and Final Paragraph of 8 speakers

\begin{tabular}{|l|l|l|l|l|l|l|l|l|}
\hline \multicolumn{8}{|c|}{ Correlation } \\
\hline Paragraph & SP1 & SP2 & SP3 & SP4 & SP5 & SP6 & SP7 & SP8 \\
\hline Initial & 0.666 & 0.0058 & 0.144393 & 0.222037 & -0.13835 & 0.005843 & -0.04049 & 0.064 \\
\hline Intermediate & 0.93614 & 0.193856 & 0.049007 & 0.239726 & 0.129241 & 0.362003 & 0.035346 & 0.005 \\
\hline End & 0.282068 & -0.05442 & 0.119878 & -0.04535 & -0.08544 & 0.044086 & 0.225506 & 0.763 \\
\hline Max-Value & 0.93614 & 0.193856 & 0.144393 & 0.239726 & 0.129241 & 0.362003 & 0.225506 & 0.76307 \\
\hline
\end{tabular}

For 8 speakers database used the value of correlation is high for 5 instances out of 8 for intermediate paragraph, twice for End Paragraph and once for initial paragraph. Thus the percentage of matching pattern of silences is $87.5 \%$ for intermediate paragraph and end paragraph considered together.

\section{Conclusion}

The analysis and correlation of the data experimented for above 8 speakers' shows that the similarity of the pauses is on the higher side in the intermediate paragraph and end paragraph than in the initial paragraph. Also the pattern of taking pause of each speaker is unique and can be used to identify the speaker. This observation allows us to conclude that the natural manner of a speaker may be reflected after speaking a few sentences in the beginning. This experimentation would lead the researchers to explore this alternative approach of obtaining silence as a unique feature of a speaker for identifying a speaker.

1) The performance of the system is dependent on the speakers fluency, command over the language, the time when both recordings are done, the environment of recording and many more factors.

2) The matching percent can improve by Multi-session recording instead of single session as this might help reduce the intra-speaker variability.

3) Experimentation can be done by varying the duration of recordings, a larger paragraph could be selected which would give a database of more than the current one of $50 \mathrm{sec}$.

4) Proper mechanism to set a threshold for identifying the silence in the speech can be devised instead of the trial based or permutation combination approach. 
International Journal of Security and Its Applications

Vol. 10, No. 5 (2016)

\section{References}

[1] M. A. Anusuya and S.K. Katti, "Speech Recognition by Machine: A Review”, International journal of computer science and Information Security, (2009). 